ALEA, Lat. Am. J. Probab. Math. Stat. 15, 571-585 (2018)

DOI: $10.30757 /$ ALEA.v15-23

\title{
The contact process as seen from a random walk
}

\section{Stein Andreas Bethuelsen}

Technische Universität München

Boltzmannstr. 3, 85748 Garching, Germany.

E-mail address: stein.bethuelsen@tum.de

URL: http://ww-m14.ma.tum.de/en/people/bethuelsen

\begin{abstract}
We consider a random walk on top of the supercritical contact process. In particular, we focus on the "contact process as seen from the random walk". Under the assumption that the infection rate of the contact process is large or the jump rate of the random walk is small, we show that this process has at most two extremal measures. Moreover, the convergence to these extremal measures is characterised by whether the contact process survives or dies out, similar to the complete convergence theorem known for the ordinary contact process. Using this, we prove a law of large numbers for the random walk. Our arguments apply to the processes considered on $\mathbb{Z}^{d}, d \geq 1$, and under general assumptions on the jump probabilities of the random walk.
\end{abstract}

\section{Introduction and main results}

1.1. Motivation, background and outline. In this paper we study a random walk on top of the contact process on $\mathbb{Z}^{d}$ with $d \geq 1$. That is, we assume that the transition kernel of the random walk depends on the contact process in a local neighbourhood around the position of the random walk. This is an example of a random walk in a dynamic random environment (abbreviated by RWDRE), a class of models that have recently been the subject of intensive studies in the mathematical literature (see e.g. Andres et al., 2018, Avena et al., 2016, Birkner et al., 2016, Deuschel et al., 2017+, Hilário et al., 2015, Huveneers and Simenhaus, 2015, Redig and Völlering, 2013).

The contact process is a classical interacting particle system. This model was first introduced by Harris (1974) in the 1970's as a model for the spread of an infection in a population. In this model, an "infection" spread from one site to a neighbouring site at a constant rate $\lambda$ and a site becomes "healthy" at a constant rate 1.

Received by the editors July 18th, 2016; accepted September 3rd, 2017.

2010 Mathematics Subject Classification. 60K35, 60K37, 60F15.

Key words and phrases. Random walks in dynamic random environment, Contact process, Complete convergence theorem, Law of large numbers. 
On the one hand, the contact process is a model to which many of the mathematical tools developed for studying disordered systems apply, such as monotonicity, duality, renormalisation and coupling, and by now much is known. For instance, a full understanding of its dependence on the initial state is known by the complete convergence theorem (which we recall in Subsection 1.2.1). As a general reference about the contact process, we refer to Liggett (1999, Chapter 1).

On the other hand, the contact process is a complicated model. Indeed, since infections spread in space and time, it has a non-trivial spatial and temporal correlation structure. Moreover, the contact process has a phase transition. For infection rate $\lambda$ sufficiently small, the whole population eventually becomes healthy, irrespectively of the initial configuration. Interestingly, for infection rate above a certain threshold, infections may spread for all times with positive probability. In particular, in this regime (called the supercritical regime), the evolution of the contact process depends strongly on the initial configuration.

The contact process is also an example of a model which, in the supercritical regime, does not fall into the class of well behaved models characterised by the cone mixing condition. In particular, the general results obtained by Avena, den Hollander and Redig (2011) and Redig and Völlering (2013) do not apply to random walks on the supercritical contact process. Despite much progress in the last years, no general theory has so far been developed for RWDRE models when the dynamic random environment is not cone mixing.

For the above reasons, the study of a random walk on the supercritical contact process in the context considered in this paper was initiated by den Hollander and dos Santos (2014). They considered a class of nearest neighbour random walks on the one dimensional contact process. Combining monotonicity properties of the contact process and the random walk, they proved a law of large numbers, valid throughout the supercritical regime, and, assuming large enough infection rate, a central limit theorem. Since then, the model has been studied in several papers. We mention in particular Bethuelsen and Heydenreich (2017), who proved a law of large numbers for a version of the model on $\mathbb{Z}^{d}$ with $d \geq 1$, and Mountford and Vares (2015), who improved the central limit theorem of den Hollander and dos Santos (2014) and proved that it holds throughout the supercritical regime. See also Bethuelsen and Völlering (2016) and Birkner, Černý and Depperschmidt (2016) for related results.

In contrast to Bethuelsen and Heydenreich (2017), den Hollander and dos Santos (2014) and Mountford and Vares (2015), who studied the evolution of the random walk directly, the focus of this paper is on the "contact process as seen from the random walk"-process (abbreviated by CPSRW). That is, we study the shift-perturbed version of the ordinary contact process on $\mathbb{Z}^{d}, d \geq 1$, such that the random walk always remains at the origin. In particular, we are interested in the set of invariant measures for the CPSRW process and its convergence towards the extremal ones.

Our main contribution is that, when the infection rate of the contact process is large or the jump rate of the random walk is small, then the CPSRW process satisfies a complete convergence theorem similar to what is known for the ordinary contact process. That is, the CPSRW process has (at most) two extremal invariant measures making the process ergodic and it converges towards a mixture of these states (in the Cesàro sense) depending only on whether the underlying contact process survives or dies out. As a consequence of this result about the CPSRW process, we also 
derive limiting properties about the random walk itself. In particular, we show that it satisfies a law of large numbers under rather general assumptions on its transition kernels.

Outline of the paper. In the next subsection we give a more precise definition of our model and in particular the CPSRW process. Our main results are presented in Subsection 1.3. As preparations for the proofs, we provide in Section 2 some preliminary results about the contact process and in Section 3 we provide a particular coupling construction of our model. Section 4 contains the proofs of our main results.

\subsection{The model.}

1.2.1. The contact process. Let $\Omega=\{0,1\}^{\mathbb{Z}^{d}}$. For $\eta \in \Omega$ and $x \in \mathbb{Z}^{d}$, we denote by $\eta_{x}$ the configuration which is identical to $\eta$ except at site $x$, where a 1 is replaced by a 0 and vice versa. We also denote by $s(\eta, x):=\sum_{y \sim x} \eta(y)$, where $\sum_{y \sim x}$ is the summation over nearest neighbours of $x$.

The contact process $\left(\eta_{t}\right)_{t \geq 0}$ on $\mathbb{Z}^{d}$ with "infection" rate $\lambda>0$ and "recovery" rate 1 is the Markov process on $\Omega$ with generator $L: \mathcal{C}(\Omega ; \mathbb{R}) \mapsto \mathcal{C}(\Omega ; \mathbb{R})$, where $\mathcal{C}(\Omega ; \mathbb{R})$ denotes the space of bounded continuous functions from $\Omega$ to $\mathbb{R}$, and $L$ is given by

$$
L f(\eta)=\sum_{x \in \mathbb{Z}^{d}}\left[\eta(x)\left[f\left(\eta_{x}\right)-f(\eta)\right]+\lambda s(\eta, x)(1-\eta(x))\left[f\left(\eta_{x}\right)-f(\eta)\right]\right]
$$

We denote the semi-group generated by $L$ by $\left(S_{t}\right)_{t \geq 0}$, also considered on the space $\mathcal{C}(\Omega, \mathbb{R})$. Note that the contact process is translation invariant, that is,

$$
\mathbb{P}_{\eta, \lambda}\left(\theta_{x} \eta_{t} \in \cdot\right)=\mathbb{P}_{\theta_{x} \eta, \lambda}\left(\eta_{t} \in \cdot\right)
$$

Here $\theta_{x}$ denotes the shift operator $\theta_{x} \eta(y)=\eta(y+x)$, and $\mathbb{P}_{\eta, \lambda}$ is the path-space measure of the contact process on $D_{\Omega}[0, \infty)$, the set of càdlàg functions on $[0, \infty)$ taking values on $\Omega$, started from $\eta_{0}=\eta$ and with parameter $\lambda$. Further, denote by $\mathcal{F}$ the product $\sigma$-algebra corresponding to $\Omega$ and let $\mathcal{M}_{1}(\Omega)$ denote the set of probability measures on $(\Omega, \mathcal{F})$. By $\delta_{\eta} \in \mathcal{M}_{1}(\Omega)$ we denote the measure which concentrates on $\eta \in \Omega$. For $\mu \in \mathcal{M}_{1}(\Omega)$ we denote by $\mathbb{P}_{\mu, \lambda}$ the path-space measure of $\left(\eta_{t}\right)_{t \geq 0}$ when the contact process is started from $\mu$, that is, $\mathbb{P}_{\mu, \lambda}(\cdot):=\int_{\Omega} \mathbb{P}_{\eta, \lambda}(\cdot) \mu(d \eta)$.

The empty configuration where the state of every site is equal to 0 , denoted by $\overline{0}$, is an absorbing state for the contact process since $s(\overline{0}, x)=0$ for all $x \in \mathbb{Z}^{d}$. On the other hand, when started from the configuration where the state of every site is equal to 1 , denoted by $\overline{1}$, the contact process is known to evolve towards an equilibrium measure called the upper invariant measure. We denote this measure by $\bar{\nu}_{\lambda}$.

As already mentioned, the contact process has a phase transition. That is, there is a critical threshold $\lambda_{c} \in(0, \infty)$, where $\lambda_{c}$ depends on the dimension, such that $\bar{\nu}_{\lambda}=\delta_{\overline{0}}$ for $\lambda \in\left(0, \lambda_{c}\right]$ and, for all $\lambda \in\left(\lambda_{c}, \infty\right)$, it holds that $\bar{\nu}_{\lambda}(\eta(x)=1)>0$ for any $x \in \mathbb{Z}^{d}$. Further, the two measures, $\delta_{\overline{0}}$ and $\bar{\nu}_{\lambda}$, are the only extremal measures for the contact process on $\mathbb{Z}^{d}$. A complete description of the convergence towards any mixture of them is known by the complete convergence theorem, which for later reference we state next. (For a proof we refer to Liggett, 1999, Theorem 1.2.27). 
Theorem 1.1 (Complete Convergence for $\left(\eta_{t}\right)$ ). Let $\tau:=\inf \left\{t \geq 0: \eta_{t}=\overline{0}\right\}$. Then, for $\lambda>0$ and $\eta \in \Omega$;

$$
\delta_{\eta} S_{t} \Longrightarrow \mathbb{P}_{\eta, \lambda}(\tau<\infty) \delta_{\overline{0}}+\mathbb{P}_{\eta, \lambda}(\tau=\infty) \bar{\nu}_{\lambda} \quad \text { as } t \rightarrow \infty,
$$

where $\Longrightarrow$ denotes weak convergence.

1.2.2. The random walk. The random walk $\left(X_{t}\right)$ is a process on $\mathbb{Z}^{d}$ whose transition probabilities depend on the state of the contact process in a neighbourhood around the random walk. More precisely, we assume (w.l.o.g.) that $X_{0}=o$, where $o \in \mathbb{Z}^{d}$ denotes the origin. Further, at any time $t>0$, the rate to jump from site $x$ to site $x+z$, given that the contact process is in state $\eta$ at time $t$, is given by $\gamma \alpha\left(\theta_{x} \eta, z\right) \in[0, \infty)$. Here, $\gamma \in[0, \infty)$ is a parameter of the model.

In order for the above process to be well defined, we need to pose some regularity assumptions. For this purpose, we assume throughout this paper that

$$
\|\alpha\|_{1}:=\sum_{z \in \mathbb{Z}^{d}}\|z\| \sup _{\eta \in \Omega}|\alpha(\eta, z)|<\infty,
$$

and that for some $R \in \mathbb{N}$ and every $z \in \mathbb{Z}^{d}$;

$$
\alpha(\eta, z)-\alpha(\omega, z)=0 \text { whenever } \eta \equiv \omega \text { on }[-R, R]^{d} .
$$

Assumption (1.4) assures that the position of $\left(X_{t}\right)$ has a first moment, whereas Assumption (1.5) says that the random walk only depends on the contact process within a finite region around its location. Note that the jump rate of $\left(X_{t}\right)$ is bounded by $\gamma\|\alpha\|_{1}$.

Further, we say that the random walk is elliptic if there is a finite subset $E=$ $\left\{e_{1}, \ldots, e_{n}\right\}$ of $\mathbb{Z}^{d}$ such that

$$
\alpha\left(\eta, e_{i}\right)>0 \quad \forall \eta \in \Omega \text { and } i \in\{1, \ldots, n\},
$$

and such that $\alpha(\eta, y)>0$ for some $\eta \in \Omega$ and $y \in \mathbb{Z}^{d}$ if and only if $y=\sum_{i=1}^{n} a_{i} e_{i}$ with $a_{i} \in\{0,1,2, \ldots\}, i=1, \ldots, n$.

Lastly, for $\xi_{[0, \infty)} \in D_{\Omega}[0, \infty)$, let $P^{\xi_{[0, \infty)}}$ denote the quenched law of $\left(X_{t}\right)$ in environment $\xi_{[0, \infty)}$. For $\mu \in \mathcal{M}_{1}(\Omega)$, the annealed law of $\left(X_{t}\right)$ is given by

$$
P^{\mu}(\cdot):=\int_{D_{\Omega}[0, \infty)} P^{\xi_{[0, \infty)}(\cdot) \mathbb{P}_{\mu, \lambda}\left(d \xi_{[0, \infty)}\right) .}
$$

If $\mu=\delta_{\eta}$ for some $\eta \in \Omega$ we write $P^{\eta}$ for $P^{\delta_{\eta}}$.

1.2.3. The contact process as seen from a random walk. "The contact process seen from the random walk" (that is, the CPSRW process) is the key object of this paper. This process, which is also useful for understanding the asymptotic behaviour of the random walk itself, is the Markov process on $\Omega$ with generator

$$
L^{E P} f(\eta):=L f(\eta)+\gamma \sum_{z \in \mathbb{Z}^{d}} \alpha(\eta, z)\left[f\left(\theta_{-z} \eta\right)-f(\eta)\right]
$$

corresponding semigroup $\left(S_{t}^{E P}\right)$, both acting on $\mathcal{C}(\Omega ; \mathbb{R})$, and with path-space measure denoted by $\mathbb{P}_{\eta, \lambda}^{E P}$. Here, the superscript EP is an abbreviation for environment process and is used to distinguish it from $\mathbb{P}_{\eta, \lambda}$, the path-space measure of the contact process. 
1.3. Main theorems. As for the ordinary contact process, it is clear that $\overline{0}$ is an absorbing state for the CPSRW process as well. If $\lambda<\lambda_{c}$ it is not difficult to show that $\delta_{\overline{0}}$ is the only stationary distribution for $\left(\eta_{t}^{E P}\right)$. This follows for instance from the methods developed in Redig and Völlering (2013) together with well known convergence estimates towards $\overline{0}$ for the subcritical contact process, see Liggett (1999, Theorem 1.2.48).

On the other hand, when $\lambda>\lambda_{c}$, one can often show that there exists more than one stationary distribution for the CPSRW process. For this, it is sufficient to show that there is a site $x \in \mathbb{Z}^{d}$ and an $\eta \in \Omega$ such that,

$$
\mathbb{P}_{\eta, \lambda}^{E P}\left(\liminf _{t \rightarrow \infty} \frac{1}{t} \int_{0}^{t} \eta_{s}^{E P}(x) d s>0\right)>0 .
$$

That (1.9) holds when $\lambda>\lambda_{c}$ can been shown by several methods. For instance, Bethuelsen and Heydenreich (2017, Theorem 1.4) and den Hollander and dos Santos (2014, Theorem 1), both proven via monotonicity arguments and particular properties of the contact process, imply that (1.9) holds for the class of models studied in these papers. In dos Santos (2014) another method is put forward, by use of multiscale analysis, and applied to a random walk on the exclusion process. This method can presumably be applied to random walks on the contact process as well.

Ideally we would like to describe the entire class of stationary distributions corresponding to $\left(\eta_{t}^{E P}\right)$, given the transition kernel of $\left(X_{t}\right)$ and the infection parameter $\lambda$. As we saw in Theorem 1.1, a complete description is at hand for the ordinary contact process, i.e. when not perturbed by the random walk. Our main theorem shows that a similar statement holds for $\left(\eta_{t}^{E P}\right)$ when either $\lambda$ is sufficiently large or $\gamma$ is sufficiently small.

Theorem 1.2 (Complete convergence for $\left.\left(\eta_{t}^{E P}\right)\right)$. Assume that $\left(X_{t}\right)$ satisfies Assumptions (1.4) and (1.5), and that it is elliptic.

a: Let $\lambda \in\left(\lambda_{c}, \infty\right)$. Then there is a $\gamma_{0} \in(0, \infty)$ such that for all $\gamma<\gamma_{0}$ there exists $\bar{\nu}_{\lambda}^{E P} \in \mathcal{M}_{1}(\Omega)$ making $\mathbb{P}_{\bar{\nu}_{\lambda}^{E P}, \lambda}^{E P}$ stationary and ergodic with respect to time-shifts. Furthermore, for any $\eta \in \Omega$,

$$
t^{-1} \int_{0}^{t} \delta_{\eta} S_{s}^{E P} d s \Longrightarrow \mathbb{P}_{\eta, \lambda}(\tau=\infty) \bar{\nu}_{\lambda}^{E P}+\mathbb{P}_{\eta, \lambda}(\tau<\infty) \delta_{\overline{0}} \quad \text { as } t \rightarrow \infty .
$$

b: Let $\gamma \in(0, \infty)$. Then there is a $\lambda_{0} \in(0, \infty)$ such that for all $\lambda>\lambda_{0}$ there exists $\bar{\nu}_{\lambda}^{E P} \in \mathcal{M}_{1}(\Omega)$ making $\mathbb{P}_{\bar{\nu}_{\lambda}^{E P}, \lambda}^{E P}$ stationary and ergodic with respect to time-shifts. Furthermore, for any $\eta \in \Omega$,

$$
t^{-1} \int_{0}^{t} \delta_{\eta} S_{s}^{E P} \Longrightarrow \mathbb{P}_{\eta, \lambda}(\tau=\infty) \bar{\nu}_{\lambda}^{E P}+\mathbb{P}_{\eta, \lambda}(\tau<\infty) \delta_{\overline{0}} \quad \text { as } t \rightarrow \infty .
$$

The choice of $\gamma_{0}$ and $\lambda_{0}$ in Theorem 1.2 is related to the asymptotic speed at which an infection spreads. That is, we require the random walk trajectory to eventually be contained inside a forward space-time cone in which, for any starting configuration $\eta \in \Omega \backslash\{\overline{0}\}$, the contact process conditioned on survival is approximately in equilibrium. This is similar in spirit to the assumption on $\lambda$ in den Hollander and dos Santos (2014, Theorem 2). 
Similar perturbative regimes have recently been studied for several other RWDRE models with non-uniform dependence on the initial configuration, in particular by Avena, Blondel and Faggionato (2016), Hilário, den Hollander, dos Santos, Sidoravicius and Teixeira (2015) and Huveneers and Simenhaus (2015). These very interesting works do not overlap with that of this paper and are furthermore based on very different methods.

The proof of Theorem 1.2 follows by a coupling argument and uses known mixing properties of the supercritical contact process together with basic ergodic theory, and does not (directly) rely on the monotonicity properties of the contact process. For what appears to be only due to technical matters, we restrict to convergence in the sense of Cesàro.

We further note that the strategy of the proof can be applied to other models with similar mixing properties as the contact process. For instance, Theorem 1.2 can be shown to hold for certain extensions of our model where the random walk is allowed to interact with the medium, i.e. the contact process, by locally adding/removing infections. Such extensions may be natural from an application point of view.

The ellipticity assumption in Theorem 1.2 seems necessary for the theorem to hold in general. Indeed, an example of a non-elliptic random walk for which there exists three extremal invariant measures for $\left(\eta_{t}^{E P}\right)$ can be constructed by making the random walk resemble the behaviour of the rightmost particle process of the contact process on $\mathbb{Z}$.

One way to achieve this is by considering a random walk that jumps deterministically to the right at a rate $\gamma \geq \lambda$ when on an infected site and otherwise as a simple random walk with jump rate 1 . Considering the corresponding CPSRW process started from $\overline{0}, \overline{1}$ or the configuration where all sites on the negative integers are infected and the remaining sites are healthy, it is not difficult to show (using results about the distribution of the contact process seen from the rightmost particle, e.g. Galves and Presutti, 1987) that this process has three invariant measures, all singular with respect to the other two.

Presumably, a similar reasoning can be made rigorous when $\lambda$ is close to the critical value or $\gamma^{-1}$ is close to 0 , even in cases where $\left(X_{t}\right)$ is elliptic. On the other hand, for the case considered in Theorem 1.2, we do not think the ellipticity assumption is really necessary. We prove this rigorously in the case the contact process is started from $\bar{\nu}_{\lambda}$, as stated next.

Theorem 1.3 (Convergence of the upper invariant measure). Assume that $\left(X_{t}\right)$ satisfies Assumptions (1.4) and (1.5). Furthermore, let $\lambda$ and $\gamma$ be as in Theorem 1.2. Then there exists $\bar{\nu}_{\lambda}^{E P} \in \mathcal{M}_{1}(\Omega)$ making $\mathbb{P}_{\bar{\nu}_{\lambda}^{E P}, \lambda}^{E P}$ stationary and ergodic with respect to time-shifts and such that

$$
t^{-1} \int_{0}^{t} \bar{\nu}_{\lambda} S_{s}^{E P} d s \Longrightarrow \bar{\nu}_{\lambda}^{E P} \in \mathcal{M}_{1}(\Omega) \quad \text { as } t \rightarrow \infty .
$$

As mentioned above, under fairly general assumptions on the random walk and assuming that either $\lambda$ is large or $\gamma$ is small, we believe that the CPSRW-process has exactly two extremal measures. That is, in Theorems 1.2 and 1.3 , we have $\bar{\nu}_{\lambda}^{E P} \neq \delta_{\overline{0}}$. We do not provide a proof of this here. Nevertheless, from the ergodic properties of $\bar{\nu}_{\lambda}^{E P}$ only, we infer information about the random walk. 
Theorem 1.4 (Law of large numbers). Under the assumptions of Theorem 1.2 there exists $v_{0}, v_{1} \in \mathbb{R}^{d}$ such that for all $\eta \in \Omega$,

$$
\lim _{t \rightarrow \infty} t^{-1} X_{t}=\mathbb{P}_{\eta, \lambda}(\tau<\infty) v_{0}+\mathbb{P}_{\eta, \lambda}(\tau=\infty) v_{1}, \quad P^{\eta}-\text { a.s. }
$$

Relaxing the ellipticity assumption on $\left(X_{t}\right),(1.13)$ holds $P^{\bar{\nu}_{\lambda}}-a . s$.

Remark 1.5. Note that $\mathbb{P}_{\eta, \lambda}(\tau=\infty)=1$ if and only if $\eta$ has infinitely many infections, as follows by Liggett (1999, Theorem 2.30). In particular, the limit in (1.13) equals $v_{1} \in \mathbb{R}^{d}$ when the contact process is started from the upper invariant measure $\bar{\nu}_{\lambda}$.

Presumably, the law of large numbers in Theorem 1.4 can be extended to a functional central limit theorem under the annealed law. For this, from the existence of $\bar{\nu}_{\lambda}^{E P}$ that is ergodic under $\left(\eta_{t}^{E P}\right)$, martingale methods (as used e.g. in Redig and Völlering, 2013) seem useful.

To this end, a remark about the critical case (i.e., when $\lambda=\lambda_{c}$ ) is in place. In this case, $\bar{\nu}_{\lambda_{c}}=\delta_{\overline{0}}$, as was proven by Bezuidenhout and Grimmett (1990). This result has since been extended to several models. On the other hand, still for $\lambda=\lambda_{c}$, the contact process process as seen from the rightmost particle is known to have a non-trivial invariant measure, as shown in Cox, Durrett and Schinazi (1991).

By using Bethuelsen and Völlering (2016, Theorem 4.5) and monotonicity of the contact process, it is not difficult to show that for $\lambda=\lambda_{c}$ any invariant measure for the CPSRW process concentrates on configurations having 0 asymptotic density. We believe that, under reasonable (ellipticity) assumptions, the CPSRW process with $\lambda=\lambda_{c}$ has no non-trivial invariant measure. However, to show this rigorously seems challenging since the critical contact process has slowly decaying space-time correlation structure.

\section{Preliminaries about the contact process}

Important to our approach is the existence of a coupling $\widehat{\mathbb{P}}_{\eta, \omega}^{\lambda}$ of the contact process started from any two $\eta, \omega \in \Omega$. The canonical choice is the graphical construction coupling, see Liggett (1999, p. 32-34), however, any other coupling satisfying (2.6) and (2.14) below will do just as fine.

For $\eta, \omega \in \Omega$, the coupled pair $\left(\eta_{t}^{1}, \eta_{t}^{2}\right)_{t \geq 0}$ denotes two copies of the contact process, started from $\eta_{0}^{1}=\eta$ and $\eta_{0}^{2}=\omega$ respectively. Recall that, by definition, a coupling has the marginals

$$
\widehat{\mathbb{P}}_{\eta, \omega}^{\lambda}\left(\eta_{t}^{1} \in \cdot\right)=\mathbb{P}_{\eta, \lambda}\left(\eta_{t} \in \cdot\right) \text { and } \widehat{\mathbb{P}}_{\eta, \omega}^{\lambda}\left(\eta_{t}^{2} \in \cdot\right)=\mathbb{P}_{\omega, \lambda}\left(\eta_{t} \in \cdot\right) .
$$

We are in this paper mainly interested in the contact process with $\lambda>\lambda_{c}$ for which $\bar{\nu}_{\lambda}$ is non-trivial. In this regime a more global description of the contact process is at hand and known as the shape theorem. For this, denote by $\left(\eta_{t}^{o}\right)$ and $\left(\eta_{t}^{\overline{1}}\right)$ the contact process started from only the origin initially infected and the entire lattice initially infected respectively and define for $t \geq 0$,

$$
\begin{aligned}
& H_{t}:=\left\{x \in \mathbb{Z}^{d}: \eta_{s}^{o}(x)=1 \text { for some } 0 \leq s \leq t\right\} ; \\
& K_{t}:=\left\{x \in \mathbb{Z}^{d}: \eta_{s}^{o}(x)=\eta_{s}^{\overline{1}}(x) \forall s \geq t\right\} .
\end{aligned}
$$

$H_{t}$ is the set of sites which have been visited by an infection by time $t$ when the contact process is started with only the origin infected at time $0 . K_{t}$ is the subset of $\mathbb{Z}^{d}$ where $\left(\eta_{t}^{o}\right)$ and $\left(\eta_{t}^{\overline{1}}\right)$ remain coupled for all time after time $t$. The next result 
shows that when $\lambda>\lambda_{c}$, then $t^{-1}\left(H_{t} \cap K_{t}\right)$ has an asymptotic shape. To state the result it is convenient to consider

$$
\bar{H}_{t}:=\bigcup_{x \in H_{t}}(x+Q) \text { and } \bar{K}_{t}:=\bigcup_{x \in K_{t}}(x+Q), \quad Q=\left[-\frac{1}{2}, \frac{1}{2}\right]^{d} .
$$

Lastly, for $\omega \in \Omega$, denote by $\tau^{\omega}:=\inf \left\{t \geq 0: \eta_{t}^{\omega}=\overline{0}\right\}$ the time until the contact process started from $\omega$ "dies out". We write $\tau^{o}:=\inf \left\{t \geq 0: \eta_{t}^{o}=\overline{0}\right\}$ for the case when $\omega(x)=1$ for $x=o$ only.

We are now prepared to state a version of the shape theorem, which in this generality follows from Garet and Marchand (2012, Theorem 3).

Theorem 2.1 (The shape theorem). Suppose $\lambda>\lambda_{c}$. There exists a convex set $D=D(\lambda) \subset \mathbb{R}^{d}$ such that, for any $\epsilon \in(0,1)$, on the event $\left\{\tau^{o}=\infty\right\}$,

$$
\lim _{T \rightarrow \infty} \widehat{\mathbb{P}}_{o, \overline{1}}^{\lambda}\left((1-\epsilon) D \subset \frac{1}{t}\left(\bar{H}_{t} \cap \bar{K}_{t}\right) \subset \frac{1}{t} \bar{H}_{t} \subset(1+\epsilon) D \forall t \geq T\right)=1 .
$$

Moreover, there is a function $f:\left(\lambda_{c}, \infty\right) \rightarrow(0, \infty)$, non-decreasing, and such that $\left\{x \in \mathbb{R}^{d}:\|x\|_{1} \leq f(\lambda)\right\} \subset D(\lambda)$ and $\lim _{\lambda \rightarrow \infty} f(\lambda)=\infty$.

Theorem 2.1 implies mixing properties for the contact process when started from other configurations than only the origin initially infected, as we show next.

Lemma 2.2. Let $\lambda>\lambda_{c}$ and consider the contact processes $\left(\eta_{t}^{\eta}\right)$ and $\left(\eta_{t}^{\overline{1}}\right)$, started from $\eta$ and $\overline{1}$ respectively, where $\eta \in \Omega \backslash\{\overline{0}\}$. Then, for any $\epsilon \in(0,1)$ and with $D$ as in Theorem 2.1,

$$
\lim _{T \rightarrow \infty} \widehat{\mathbb{P}}_{\eta, \overline{1}}^{\lambda}\left(\eta_{t}^{\eta}(x)=\eta_{t}^{\overline{1}}(x) \forall x \in t(1-\epsilon) D \forall t \geq T \mid \tau^{\eta}=\infty\right)=1 .
$$

Proof: Since the path measure of the contact process is translation invariant with respect to spatial shifts, (2.6) holds in the case when $\eta(x)=1$ for only one site $x \in \mathbb{Z}^{d}$, as follows immediately from Theorem 2.1. Indeed, for any $0<\epsilon_{1}<\epsilon_{2}$, it holds that $t\left(1-\epsilon_{2}\right) D \subset \theta_{x} t\left(1-\epsilon_{1}\right) D$ for all $t$ sufficiently large.

For $T \in(0, \infty)$, let $A_{T}=\left\{\eta_{t}^{\eta}(x) \neq \eta_{t}^{\overline{1}}(x)\right.$ for some $x \in t(1-\epsilon) D$ and $\left.t \geq T\right\}$. Fix $x_{1}, \ldots, x_{n} \in \mathbb{Z}^{d}$ and assume that $\eta \in \Omega$ is such that $\eta(y)=1$ only when $y=x_{1}, \ldots, x_{n}$. By using that the contact process is monotone and additive (in particular, that $\left.\left\{\tau^{\eta}=\infty\right\}=\cup_{i=1}^{n}\left\{\tau^{x_{i}}=\infty\right\}\right)$, we have

$$
\begin{aligned}
\widehat{\mathbb{P}}_{\eta, \overline{1}}^{\lambda}\left(A_{T} \mid \tau^{\eta}=\infty\right) & =\widehat{\mathbb{P}}_{\eta, \overline{1}}^{\lambda}\left(\tau^{\eta}=\infty\right)^{-1} \widehat{\mathbb{P}}_{\eta, \overline{1}}^{\lambda}\left(A_{T}, \tau^{\eta}=\infty\right) \\
& =\widehat{\mathbb{P}}_{\eta, \overline{1}}^{\lambda}\left(\tau^{\eta}=\infty\right)^{-1} \widehat{\mathbb{P}}_{\eta, \overline{1}}^{\lambda}\left(A_{T} \cap\left(\cup_{i=1}^{n}\left\{\tau^{x_{i}}=\infty\right\}\right)\right) \\
& \leq \widehat{\mathbb{P}}_{\eta, \overline{1}}^{\lambda}\left(\tau^{\eta}=\infty\right)^{-1} \sum_{i=1}^{n} \widehat{\mathbb{P}}_{\sigma_{i}, \overline{1}}^{\lambda}\left(A_{T} \cap\left\{\tau^{x_{i}}=\infty\right\}\right) \\
& \leq \sum_{i=1}^{n} \widehat{\mathbb{P}}_{\sigma_{i}, \overline{1}}^{\lambda}\left(A_{T} \mid\left\{\tau^{x_{i}}=\infty\right\}\right),
\end{aligned}
$$

where $\sigma_{i} \in \Omega$ is such that $\sigma_{i}(y)=1$ only if $y=x_{i}$. Observe that each term in the latter sum converges to 0 as $T \rightarrow \infty$, as follows by the first part of the proof. Hence, also $\widehat{\mathbb{P}}_{\eta, \overline{1}}^{\lambda}\left(A_{T} \mid \tau^{\eta}=\infty\right) \rightarrow 0$ as $T \rightarrow \infty$, from which we conclude that (2.6) holds when $\eta$ has finitely many 1 's. 
What remains to be shown is that (2.6) holds when the contact process is started from a configuration with infinitely many sites infected. In this case, $\tau^{\eta}=\infty$ a.s. (see Remark 1.5) and so

$$
\widehat{\mathbb{P}}_{\eta, \overline{1}}^{\lambda}\left(A_{T}^{c} \mid \tau^{\eta}=\infty\right)=\widehat{\mathbb{P}}_{\eta, \overline{1}}^{\lambda}\left(A_{T}^{c}\right) .
$$

Let $\left(N_{n}\right)_{n \in \mathbb{N}}$ be a non-decreasing sequence such that $\sum_{x \in\left[-N_{n}, N_{n}\right]^{d}} \eta(x) \geq n$ and denote by $\sigma_{n} \in \Omega$ the configuration which equals $\eta$ on $\left[-N_{n}, N_{n}\right]^{d}$ and equals 0 outside $\left[-N_{n}, N_{n}\right]^{d}$. By the monotonicity property of the graphical construction coupling we have

$$
\begin{aligned}
\widehat{\mathbb{P}}_{\eta, \overline{1}}^{\lambda}\left(A_{T}^{c}\right) \geq \widehat{\mathbb{P}}_{\sigma_{n}, \overline{1}}^{\lambda}\left(A_{T}^{c}\right) & \geq \widehat{\mathbb{P}}_{\sigma_{n}, \overline{1}}^{\lambda}\left(A_{T}^{c} \cap\left\{\tau^{\sigma_{n}}=\infty\right\}\right) \\
& =\widehat{\mathbb{P}}_{\sigma_{n}, \overline{1}}^{\lambda}\left(\tau^{\sigma_{n}}=\infty\right) \widehat{\mathbb{P}}_{\sigma_{n}, \overline{1}}^{\lambda}\left(A_{T}^{c} \mid \tau^{\sigma_{n}}=\infty\right) .
\end{aligned}
$$

Hence, taking $T \rightarrow \infty$ yields that $\lim _{T \rightarrow \infty} \widehat{\mathbb{P}}_{\eta, \overline{1}}^{\lambda}\left(A_{T}^{c}\right) \geq \widehat{\mathbb{P}}_{\eta, \overline{1}}^{\lambda}\left(\tau^{\sigma_{n}}=\infty\right)$. Taking $n \rightarrow \infty$, by Liggett (1999, Theorem 1.2.30), we conclude the proof.

We also need to control the contact process started from a finite number of 1's and conditioned on dying out, for which we have the following lemma.

Lemma 2.3. Let $\lambda>\lambda_{c}$ and consider the contact processes $\left(\eta_{t}^{\eta}\right)$ and $\left(\eta_{t}^{\overline{0}}\right)$, started from $\eta$ and $\overline{0}$ respectively, where $\eta$ satisfies $\sum_{x \in \mathbb{Z}^{d}} \eta(x)<\infty$. Then, for any $\Delta \subset$ $\mathbb{Z}^{d}$

$$
\lim _{T \rightarrow \infty} \widehat{\mathbb{P}}_{\eta, \overline{0}}^{\lambda}\left(\eta_{t}^{\eta}(x)=\eta_{t}^{\overline{0}}(x) \forall x \in t \Delta \forall t \geq T \mid \tau^{\eta}<\infty\right)=1 .
$$

Proof: This is immediate, since the left hand side of (2.14) is greater than or equal to $1-\lim _{T \rightarrow \infty} \widehat{\mathbb{P}}_{\eta, \overline{0}}^{\lambda}\left(\tau^{\eta}>T \mid \tau^{\eta}<\infty\right)=1$.

\section{Coupling construction}

Given the coupling $\widehat{\mathbb{P}}_{\eta, \omega}^{\lambda}$ of the contact process from the previous section, for each $T \in[0, \infty)$, we show in the following lemma how to extend it to a coupling $\widehat{\mathbb{P}}_{\eta, \omega, T}^{\lambda}$ also containing the evolution of two random walks $\left(X_{t}^{1}, X_{t}^{2}\right)_{t \geq 0}$ on $\left(\eta_{t}^{1}, \eta_{t}^{2}\right)_{t \geq 0}$. The coupling construction is motivated by the coupling used in den Hollander and dos Santos (2014, Section 3), and can be seen as a generalisation of their approach to general dimensions and general transition kernels.

Before stating the lemma we need to introduce some notation. For $\gamma \in(0, \infty)$, denote by $\mathcal{R}(\gamma) \subset \mathbb{R}^{d}$ the convex hull of the transition kernels of $\left(X_{t}\right)$, that is,

$$
\mathcal{R}(\gamma):=\gamma \cdot \operatorname{conv}\left(\sum_{z \in \mathbb{Z}^{d}} z \alpha(\eta, z), \eta \in \Omega\right)
$$

Further, for $\eta, \omega \in \Omega$ and $T, \epsilon>0$, let

$$
C_{T, \epsilon}(\eta, \omega):=\left\{\eta_{s}^{\eta}(x)=\eta_{s}^{\omega}(x) \forall x \in s(1+\epsilon) \mathcal{R}(\gamma)+[-R, R]^{d}, s \in[T, \infty)\right\}
$$

denote the event that the contact processes started from $\eta$ and $\omega$ respectively are perfectly coupled inside $s(1+\epsilon) \mathcal{R}(\gamma)+[-R, R]^{d} \subset \mathbb{Z}^{d}$ for all $s \geq T$, and let

$$
D_{T, \epsilon}:=\left\{X_{t}^{1}, X_{t}^{2} \in t(1+\epsilon) \mathcal{R}(\gamma) \forall t \geq T\right\} .
$$

Lemma 3.1. Let $T \in[0, \infty)$ and let $\eta, \omega \in \Omega$. There exists a coupling $\widehat{\mathbb{P}}_{\eta, \omega, T}^{\lambda}$ with the following properties: 
a: (Marginals) The coupling supports two contact processes and corresponding random walks:

1. $\widehat{\mathbb{P}}_{\eta, \omega, T}^{\lambda}\left(\left(\eta_{t}^{1}, X_{t}^{1}\right) \in \cdot\right)=\tilde{\mathbb{P}}_{\eta, \lambda}\left(\left(\eta_{t}, X_{t}\right) \in \cdot\right) ;$

2. $\widehat{\mathbb{P}}_{\eta, \omega, T}^{\lambda}\left(\left(\eta_{t}^{2}, X_{t}^{2}\right) \in \cdot\right)=\tilde{\mathbb{P}}_{\omega, \lambda}\left(\left(\eta_{t}, X_{t}\right) \in \cdot\right)$,

where $\tilde{\mathbb{P}}_{\eta, \lambda}$ is the path measure of the joint process $\left(\eta_{t}, X_{t}\right)$.

b: (Extension of $\widehat{\mathbb{P}}_{\eta, \omega}^{\lambda}$ ) The contact processes behave as under $\widehat{\mathbb{P}}_{\eta, \omega}^{\lambda}$,

$$
\widehat{\mathbb{P}}_{\eta, \omega, T}^{\lambda}\left(\left(\eta_{t}^{1}, \eta_{t}^{2}\right) \in \cdot\right)=\widehat{\mathbb{P}}_{\eta, \omega}^{\lambda}\left(\left(\eta_{t}^{1}, \eta_{t}^{2}\right) \in \cdot\right) .
$$

c: (Coupling of the walkers) The jumping times of $X_{t}^{1}$ and $X_{t}^{2}$ are independent up to time $T$ and identical after time $T$. Furthermore, for any $\epsilon \in(0,1)$,

$$
\widehat{\mathbb{P}}_{\eta, \omega, T}^{\lambda}\left(X_{t}^{1}=X_{t}^{2} \forall t \geq T \mid X_{T}^{1}=X_{T}^{2}, C_{T, \epsilon}(\eta, \omega), D_{T, \epsilon}\right)=1 .
$$

Proof: To obtain the properties listed above, we extend the original coupling $\widehat{\mathbb{P}}_{\eta, \omega}^{\lambda}$ to contain three Poisson processes $N^{i}$ with $i \in\{1,2,3\}$, all with rates $\lambda_{i}:=\gamma\|\alpha\|_{1}$, as well as a sufficient supply of independent uniform $[0,1]$ variables for each $i \in$ $\{1,2,3\}$, denoted by $U^{i}$. The Poisson processes are chosen independent of $\widehat{\mathbb{P}}_{\eta, \omega}^{\lambda}$ and thus property b) is immediate.

To obtain the properties described in a) and c), we chose the Poisson processes $N^{1}$ and $N^{3}$ independent from each other, as well as the corresponding variables $U^{1}$ and $U^{3}$. Furthermore, the process $N^{2}$ is given by

$$
N_{t}^{2}:=\left\{\begin{array}{cc}
N_{t}^{3} & \text { if } t \leq T \\
N_{T}^{3}+N_{t}^{1}-N_{T}^{1} & \text { if } t>T
\end{array}\right.
$$

and the variables $U^{2}$ are given by

$$
U_{n}^{2}:=\left\{\begin{array}{cl}
U_{n}^{3} & \text { if } n \leq N_{T}^{3} \\
U_{n+N_{T}^{1}-N_{T}^{3}}^{1} & \text { otherwise. }
\end{array}\right.
$$

Now, for $j \in\{1,2\}$, the random walk $X^{j}$ starts from $o$ and exclusively (but not necessarily) jump when the Poisson clocks $N^{j}$ rings. To make this precise, enumerate $\mathbb{Z}^{d}=\left\{z_{1}, z_{2}, z_{3}, \ldots\right\}$ and let for each $\eta \in \Omega$ and $m \in \mathbb{N}, p(\eta, m):=\sum_{i=1}^{m} \alpha\left(\eta, z_{i}\right)$. When the clock $N^{j}$ rings for the $k$ 'th time, the random walk jumps from $X_{t}^{j}$ to $X_{t}^{j}+z_{i}$ only if the uniform $[0,1]$ variable $U_{k}^{j}$ satisfies

$$
\|\alpha\|_{1}^{-1} p\left(\theta_{X_{t}^{j}} \eta_{t}^{j}, z_{m-1}\right) \leq U_{k}^{j}<\|\alpha\|_{1}^{-1} p\left(\theta_{X_{t}^{j}} \eta_{t}^{j}, z_{m}\right) .
$$

Clearly this yields property a). Furthermore, note that both the random walks use independent Poisson clocks and $U$ 's up to time $T$, and share the same Poisson clocks and $U$ 's after time $T$. Property c) follows as a consequence of this and since $\left(X_{t}\right)$ satisfies $(1.5)$.

\section{Proofs}

4.1. Coupling argument. In this subsection we present the coupling argument essential for the proofs of Theorems 1.2 and 1.3. For this, we first note that, as a simple consequence of our assumptions on the transition kernels of the random walk (recall Assumptions (1.4), (1.5) and Definition (3.1)), the following lemma holds.

Lemma 4.1. For any $\epsilon>0$ and any $\eta, \omega \in \Omega$, it holds that

$$
\lim _{T \rightarrow \infty} \widehat{\mathbb{P}}_{\eta, \omega, T}\left(X_{s}^{1}, X_{s}^{2} \in s(1+\epsilon) \mathcal{R}(\gamma) \forall s \geq T\right)=1 \text {. }
$$


With the help of the coupling construction in the previous section, together with Lemma 2.2 and Lemma 4.1, we next present a generalisation of den Hollander and dos Santos (2014, Proposition 3.3) which allows us to compare possible limiting measures of $\left(\eta_{t}^{E P}\right)$.

Proposition 4.2. Assume that $\left(X_{t}\right)$ satisfies Assumptions (1.4) and (1.5) and is elliptic. Furthermore, assume there exists $\mu^{E P} \in \mathcal{M}_{1}(\Omega)$ making $\mathbb{P}_{\mu^{E P}, \lambda}^{E P}$ stationary and ergodic with respect to time-shifts and such that $\mu^{E P} \neq \delta_{\overline{0}}$. If, for some $\epsilon>0$, $(1+\epsilon) \mathcal{R}(\gamma) \subset D$, then for every $f \in \mathcal{C}(\Omega ; \mathbb{R})$ and every $\omega \neq \overline{0}$

$$
\mathbb{P}_{\omega, \lambda}^{E P}\left(\lim _{t \rightarrow \infty} t^{-1} \int_{0}^{t} f\left(\eta_{s}^{E P}\right) d s=\mu^{E P}(f) \mid \tau^{\omega}=\infty\right)=1 .
$$

Proof: Let $\mu^{E P} \in \mathcal{M}_{1}(\Omega)$ be such that $\mathbb{P}_{\mu^{E P}, \lambda}^{E P}$ is ergodic with respect to timeshifts and $\mu^{E P} \neq \delta_{\overline{0}}$. By ergodicity, we know that there exist a set $B \in \mathcal{F}$ of full $\mu^{E P}$-measure such that for any $f \in \mathcal{C}(\Omega ; \mathbb{R})$, the set of bounded and continuous functions from $\Omega$ to $\mathbb{R}$, and $\eta \in B$;

$$
\mathbb{P}_{\eta, \lambda}^{E P}\left(\lim _{t \rightarrow \infty} t^{-1} \int_{0}^{t} f\left(\eta_{s}^{E P}\right) d s=\mu^{E P}(f)\right)=1
$$

Note that, we can assume that $\sum_{x \in \mathbb{Z}^{d}} \eta(x)=\infty$ for every $\eta \in B$. Indeed, if $\eta \in \Omega$ is such that $\sum_{x \in \mathbb{Z}^{d}} \eta(x)<\infty$, then with positive probability the contact process starting from $\eta$ dies out in finite time. In this event, $(4.3)$ converges to $f(\overline{0})$, which gives a contradiction since, by assumption, $\mu^{E P} \neq \delta_{\overline{0}}$.

Fix $\eta \in B$ and, for $n \in \mathbb{N}$, let $f: \Omega \rightarrow \mathbb{R}$ be a bounded and measurable function only depending on the configuration inside $[-n, n]^{d}$; that is, a local function. In order to prove Proposition 4.2 we will show that for any $\omega \neq \overline{0}$;

$$
\mathbb{P}_{\omega, \lambda}^{E P}\left(\lim _{t \rightarrow \infty} t^{-1} \int_{0}^{t} f\left(\eta_{s}^{E P}\right) d s=\mu^{E P}(f) \mid \tau^{\omega}=\infty\right)=1,
$$

irrespectively of the choice of $n$ and $f$. This readily implies the statement of Proposition 4.2 by standard arguments since every function $g \in \mathcal{C}(\Omega ; \mathbb{R})$ can be approximated by a sequence of local functions.

Since, by assumption, there is an $\epsilon>0$ such that $(1+\epsilon) \mathcal{R}(\gamma) \subset D$, we can find an $\epsilon_{1} \in(0, \epsilon)$ such that $\left(1+\epsilon_{1}\right) \mathcal{R}(\gamma) \subset\left(1-\epsilon_{1}\right) D$. Further, recall (3.2) and (3.3) and let

$$
\Gamma_{T}:=D_{T, \epsilon_{1}} \cap C_{T, \epsilon_{1}}(\eta, \omega) .
$$

Now, consider the coupling $\widehat{\mathbb{P}}_{\eta, \omega, T}^{\lambda}$ as constructed in Lemma 3.1 and note that, by Lemma 2.2 and Lemma 4.1,

$$
\lim _{T \rightarrow \infty} \widehat{\mathbb{P}}_{\eta, \omega, T}^{\lambda}\left(\Gamma_{T} \mid \tau^{\omega}=\infty\right)=1
$$

By the law of total expectation, by taking conditional expectation, (4.4) thus follows if we can show that

$$
\lim _{T \rightarrow \infty} \widehat{\mathbb{P}}_{\eta, \omega, T}^{\lambda}\left(\lim _{t \rightarrow \infty} t^{-1} \int_{0}^{t} f\left(\theta_{X_{s}^{2}} \eta_{s}^{2}\right) d s=\mu^{E P}(f) \mid \Gamma_{T}, \tau^{\omega}=\infty\right)=1 .
$$


Moreover, by property c) of the coupling construction in Lemma 3.1, it suffices to show that,

$$
\widehat{\mathbb{P}}_{\eta, \omega, T}^{\lambda}\left(\lim _{t \rightarrow \infty} t^{-1} \int_{0}^{t} f\left(\theta_{X_{s}^{2}} \eta_{s}^{2}\right) d s=\mu^{E P}(f) \mid \Gamma_{T}, \tau^{\omega}=\infty, X_{T}^{2}=x\right)=1
$$

for all $T>0$ and all $x \in \mathbb{Z}^{d}$ inside $\left(1+\epsilon_{1}\right) T \mathcal{R}(\gamma)$.

To this end, we employ the ellipticity assumption. For each fixed $x \in \mathbb{Z}^{d}$ inside $\left(1+\epsilon_{1}\right) T \mathcal{R}(\gamma)$, there exists an event $B_{x}$ generated by $\left(N_{[0, T]}^{1}, U_{\left[1, N_{[0, T]}^{1}\right]}^{1}\right)$ which has positive probability and such that $X_{T}^{1}=x$ on $B_{x}$. By property c) of the coupling construction and due to the ellipticity assumption, $B_{x}$ can be chosen independent of the evolution of $\left(\eta_{t}^{1}, \eta_{t}^{2}\right)$ and $\left(X_{t}^{2}\right)$. Using this property, we have that

$$
\begin{aligned}
& \widehat{\mathbb{P}}_{\eta, \omega, T}^{\lambda}\left(\lim _{t \rightarrow \infty} t^{-1} \int_{0}^{t} f\left(\theta_{X_{s}^{2}} \eta_{s}^{2}\right) d s=\mu^{E P}(f) \mid \Gamma_{T}, \tau^{\omega}=\infty, X_{T}^{2}=x\right) \\
= & \widehat{\mathbb{P}}_{\eta, \omega, T}^{\lambda}\left(\lim _{t \rightarrow \infty} t^{-1} \int_{0}^{t} f\left(\theta_{X_{s}^{2}} \eta_{s}^{2}\right) d s=\mu^{E P}(f) \mid \Gamma_{T}, \tau^{\omega}=\infty, X_{T}^{2}=x, B_{x}\right) \\
= & \widehat{\mathbb{P}}_{\eta, \omega, T}^{\lambda}\left(\lim _{t \rightarrow \infty} t^{-1} \int_{0}^{t} f\left(\theta_{X_{s}^{1}} \eta_{s}^{1}\right) d s=\mu^{E P}(f) \mid \Gamma_{T}, \tau^{\omega}=\infty, X_{T}^{2}=x, B_{x}\right)
\end{aligned}
$$

Here, the first equality holds since $B_{x}$ is independent of all the other variables. To see that the second equality holds, note that $\Gamma_{T}$ ensures that the contact processes are perfectly coupled inside the space-time region defined by $C_{T, \epsilon}(\eta, \omega)$. Furthermore, since $x$ is inside $\left(1+\epsilon_{1}\right) T \mathcal{R}(\gamma)$ and $X_{T}^{1}=X_{T}^{2}$, property c) of the coupling construction apply and yields that $X_{t}^{1}=X_{t}^{2}$ for all $t \geq T$. Lastly, there is a $t \in(0, \infty)$ such that $\left(1+\epsilon_{1}\right) s \mathcal{R}(\gamma)+[-n, n]^{d} \subset\left(1-\epsilon_{1}\right) s D$ for all $s \geq t$, and we conclude that $f\left(\theta_{X_{s}^{2}} \eta_{s}^{2}\right)=f\left(\theta_{X_{s}^{1}} \eta_{s}^{1}\right)$ for all $s \geq \max \{t, T\}$.

To conclude (4.4) and hence the proof of Proposition 4.2, we note that (4.11) equals 1 as a consequence of (4.3).

By a straightforward adaptation of the proof of Proposition 4.2, replacing $\mu^{E P}$ by $\delta_{\overline{0}}$ and using Lemma 2.3 instead of Lemma 2.2, we have the following statement.

Proposition 4.3. Assume that $\left(X_{t}\right)$ satisfies Assumptions (1.4) and (1.5) and is elliptic. Let $\omega \in \Omega$ be such that $\sum_{x \in \mathbb{Z}^{d}} \omega(x)<\infty$. Then, for every $f \in \mathcal{C}(\Omega ; \mathbb{R})$;

$$
\mathbb{P}_{\omega, \lambda}^{E P}\left(\lim _{t \rightarrow \infty} t^{-1} \int_{0}^{t} f\left(\eta_{s}^{E P}\right) d s=f(\overline{0}) \mid \tau^{\omega}<\infty\right)=1 .
$$

Following den Hollander and dos Santos (2014, Remark 3.4), the ellipticity assumption in the above argument is not necessary in the case when the contact process is started from the upper invariant measure. The following proposition is essential for the proof of Theorem 1.3.

Proposition 4.4. Assume that $\left(X_{t}\right)$ satisfies Assumptions (1.4) and (1.5). Furthermore, assume there exists $\mu^{E P} \in \mathcal{M}_{1}(\Omega)$ making $\mathbb{P}_{\mu^{E P}, \lambda}^{E P}$ ergodic with respect to time-shifts and such that $\mu^{E P} \neq \delta_{\overline{0}}$. If, for some $\epsilon>0, \mathcal{R}(\gamma)(1+\epsilon) \subset D$, then for every $f \in \mathcal{C}(\Omega ; \mathbb{R})$;

$$
\mathbb{P}_{\bar{\nu}_{\lambda}, \lambda}^{E P}\left(\lim _{t \rightarrow \infty} t^{-1} \int_{0}^{t} f\left(\eta_{s}^{E P}\right) d s=\mu^{E P}(f) \mid \tau=\infty\right)=1 .
$$


Proof: The statement follows as in the proof of Proposition 4.2, only with minor modifications which we highlight next. To adapt the proof, replace the conditioning on $X_{T}^{2}=x$ in (4.8) (and the proceeding derivations) by the event $\left\{N_{T}^{2}=0\right\}$, which implies $X_{T}^{2}=0$. Then, by stationarity of the contact process under $\bar{\nu}_{\lambda}$, $\left(X_{t+T}^{2}-X_{T}^{2}\right)_{t \geq 0}$ under $\widehat{\mathbb{P}}_{\eta, \bar{\nu}_{\lambda}, T}^{\lambda}\left(\cdot \mid N_{T}^{2}=0\right)$ has the same distribution as $\left(X_{t}^{2}\right)_{t \geq 0}$ under $\widehat{\mathbb{P}}_{\eta, \bar{\nu}_{\lambda}, T}^{\lambda}(\cdot)$. Since $N_{T}^{1}=0$ implies $X_{T}^{1}=0$ and has positive probability, the claim follows as in the proof of Proposition 4.2 by replacing $B_{x}$ by $\left\{N_{T}^{1}=0\right\}$.

\subsection{Proof of Theorems 1.2 and 1.3.}

Proof of Theorem 1.2: As mentioned in the introduction, the measure $\delta_{\overline{0}}$ is trivially an invariant measure for the CPSRW process. Furthermore, it clearly makes $\mathbb{P}_{\delta_{\overline{0}}, \lambda}^{E P}$ ergodic with respect to time-shifts and is hence extremal. Thus, in the (unlikely) scenario that $\delta_{\overline{0}}$ is the unique invariant measure for the CPSRW process, Theorem 1.2 follows by classical ergodic theory with $\bar{\nu}_{\lambda}^{E P}=\delta_{\overline{0}}$.

To complete the argument of Theorem 1.2, we next consider the (more likely) scenario that there exists a measure $\mu^{E P} \in \mathcal{M}_{1}(\Omega)$ invariant under $\left(\eta_{t}^{E P}\right)$ and such that $\mu^{E P} \neq \delta_{\overline{0}}$. Without loss of generality, assume that $\mu^{E P}$ is extremal and hence singular with respect to $\delta_{\overline{0}}$. By Proposition 4.2 together with Remark 1.5, the statement of Theorem 1.2 follows immediately when starting the CPSRW process with a configuration having infinitely many 1's in the case that $\mathcal{R}(\gamma)(1+\epsilon) \subset D$. In this case the CPSRW process converges towards $\bar{\nu}_{\lambda}^{E P}:=\mu^{E P}$. For fixed $\lambda>\lambda_{c}$, the statement of Theorem 1.2a thus follows by taking $\gamma$ sufficiently small. Similarly, for fixed $\gamma \in(0, \infty)$, the statement of Theorem $1.2 \mathrm{~b}$ follows by taking $\lambda$ sufficiently large, since $D=D(\lambda)$ is growing towards the whole lattice as $\lambda$ increases.

Similarly, if the starting configuration $\eta \neq \overline{0}$ has only finitely many 1 's, the CPSRW process converges towards $\bar{\nu}_{\lambda}^{E P}:=\mu^{E P}$ on the event that $\{\tau=\infty\}$. This follows again by applying Proposition 4.2. On the other hand, on the event $\{\tau<\infty\}$, by Proposition 4.3, the CPSRW process converges towards $\delta_{\overline{0}}$. This concludes the proof.

Proof of Theorem 1.3: This follows analogously to the proof of Theorem 1.2 for the case when $\eta \in \Omega$ has infinitely many 1's, by applying Proposition 4.4 instead of Proposition 4.2.

\subsection{Proof of Theorem 1.4.}

Proof of Theorem 1.4: Recall the coupling $\widehat{\mathbb{P}}_{\eta, \omega, T}^{\lambda}$ in Lemma 3.1. Fix $T \geq 0$ and note that, starting from $\eta \in \Omega$, we can represent the random walk as a function of the environment process (recall (3.6)) as follows:

$$
X_{t}:=X_{t}^{1}=\sum_{k \geq 1} \sum_{i \geq 1} z_{i} \mathbb{1}_{\left.\left\{\|\alpha\|_{1}^{-1} p\left(\eta_{J_{k}}^{E P}, z_{i-1}\right) \leq U_{k}^{1}<\|\alpha\|_{1}^{-1} p\left(\eta_{J_{k}}^{E P}, z_{i}\right)\right)\right\}} \mathbb{1}_{\left\{J_{k} \leq T\right\}},
$$

where $\left(J_{k}\right)$ denotes the jump times of $\left(N_{t}^{1}\right)$ and $\eta_{J_{k}}^{E P}=\theta_{X_{J_{k}}} \eta_{J_{k}}^{1}$. In particular, note that $X_{t}$ is a local function of the environment process since, for each $m \in \mathbb{N}$ and $\eta \in \Omega, p(\eta, m):=\sum_{i=1}^{m} \alpha\left(\eta, z_{i}\right)$ only depends on the values of $\eta$ on $[-R, R]^{d}$. Further, since $\|\alpha\|_{1}<\infty$, we have that $\sup _{s \in(0,1)} \tilde{\mathbb{E}}_{\eta, \lambda}\left[\left\|X_{s+t}-X_{t}\right\|_{1}\right]<\infty$, and 
thus

$$
\begin{aligned}
\lim _{t \rightarrow \infty} t^{-1} X_{t} & =\lim _{t \rightarrow \infty} t^{-1}\left(X_{t}-X_{\lfloor t\rfloor}\right)+\lim _{t \rightarrow \infty} t^{-1} \sum_{j=1}^{\lfloor t\rfloor} X_{j}-X_{j-1} \\
& =\lim _{t \rightarrow \infty} t^{-1} \sum_{j=1}^{\lfloor t\rfloor} X_{j}-X_{j-1} .
\end{aligned}
$$

Now, assume that $\left(X_{t}\right)$ satisfies Assumptions (1.4) and (1.5), and let $\gamma, \lambda \in(0, \infty)$ be such that either Theorem 1.2a or Theorem 1.2b hold. Consider $\left(\eta_{t}^{E P}\right)$ started from $\bar{\nu}_{\lambda}^{E P}$ for which the process is ergodic. Then the sequence $\left(X_{i}-X_{i-1}\right)_{i \geq 1}$ forms a stationary sequence and thus, by applying the ergodic theorem to (4.16), we obtain that

$$
\lim _{t \rightarrow \infty} t^{-1} X_{t}=\tilde{\mathbb{E}}_{\bar{\nu}_{\lambda}^{E P}, \lambda}\left[X_{1}\right], \quad \tilde{\mathbb{P}}_{\bar{\nu}_{\lambda}^{E P}, \lambda} \text {-a.s. }
$$

From this we conclude that (1.13) holds when the process is started from $\bar{\nu}_{\lambda}^{E P}$ with

$$
v_{1}:=\tilde{\mathbb{E}}_{\bar{\nu}_{\lambda}^{E P}, \lambda}\left[X_{1}\right]=\gamma \int \sum_{z \in \mathbb{Z}^{d}} z \alpha(\eta, z) \bar{\nu}_{\lambda}^{E P}(d \eta) \quad \text { and } v_{0}:=\gamma \sum_{z \in \mathbb{Z}^{d}} z \alpha(\overline{0}, z)
$$

If $\left(\eta_{t}^{E P}\right)$ is uniquely ergodic, then the use of the ergodic theorem above holds for the process started from any $\eta \in \Omega$, and thus we conclude (1.13) in this case with $v_{1}=v_{0}$. Next, assume $\bar{\nu}_{\lambda}^{E P} \neq \delta_{\overline{0}}$ and consider $\left(\eta_{t}^{E P}\right)$ started from a fixed (but arbitrary) $\eta \in \Omega \backslash\{\overline{0}\}$. If $\left(X_{t}\right)$ in addition is elliptic, then we have that

$$
\lim _{t \rightarrow \infty} t^{-1} X_{t}=v_{1}, \quad \tilde{\mathbb{P}}_{\eta, \lambda}\left(\cdot \mid \tau^{\eta}=\infty\right) \text {-a.s. }
$$

Indeed, this follows by the same argument as in the proof of Proposition 4.2, replacing $f\left(\theta_{X_{s}^{2}} \eta_{s}^{2}\right)$ and $f\left(\theta_{X_{s}^{1}} \eta_{s}^{1}\right)$ by $X_{s}^{2}$ and $X_{s}^{1}$ respectively, and $\mu^{E P}(f)$ by $v_{1}$ throughout the argument. By an analogous adaptation of Proposition 4.3, we also conclude that

$$
\lim _{t \rightarrow \infty} t^{-1} X_{t}=v_{0}, \quad \tilde{\mathbb{P}}_{\eta, \lambda}\left(\cdot \mid \tau^{\eta}<\infty\right) \text {-a.s. }
$$

From (4.19) and (4.20) we conclude that (1.13) holds for every $\eta \in \Omega$ in the case that $\left(X_{t}\right)$ is elliptic. It follows, by doing the same adaptation as above to the proof of Proposition 4.4, that the ellipticity assumption can be relaxed when $\left(\eta_{t}^{E P}\right)$ is started from $\bar{\nu}_{\lambda}$, and by this the proof of Theorem 1.4 is complete.

Acknowledgement. The author thanks Matthias Birkner and the referee for comments and suggestions which helped improve the presentation of this paper.

\section{References}

S. Andres, A. Chiarini, J.-D. Deuschel and M. Slowik. Quenched invariance principle for random walks with time-dependent ergodic degenerate weights. Ann. Probab. 46 (1), 302-336 (2018). MR3758732.

L. Avena, O. Blondel and A. Faggionato. A class of random walks in reversible dynamic environments: antisymmetry and applications to the East model. $J$. Stat. Phys. 165 (1), 1-23 (2016). MR3547832. 
L. Avena, F. den Hollander and F. Redig. Law of large numbers for a class of random walks in dynamic random environments. Electron. J. Probab. 16, no. 21, 587-617 (2011). MR2786643.

S. A. Bethuelsen and M. Heydenreich. Law of large numbers for random walks on attractive spin-flip dynamics. Stochastic Process. Appl. 127 (7), 2346-2372 (2017). MR3652417.

S. A. Bethuelsen and F. Völlering. Absolute continuity and weak uniform mixing of random walk in dynamic random environment. Electron. J. Probab. 21, Paper No. 71, 32 (2016). MR3580037.

C. Bezuidenhout and G. Grimmett. The critical contact process dies out. Ann. Probab. 18 (4), 1462-1482 (1990). MR1071804.

M. Birkner, J. Černý and A. Depperschmidt. Random walks in dynamic random environments and ancestry under local population regulation. Electron. J. Probab. 21, Paper No. 38, 43 (2016). MR3508685.

J. T. Cox, R. Durrett and R. Schinazi. The critical contact process seen from the right edge. Probab. Theory Related Fields 87 (3), 325-332 (1991). MR1084333.

J.-D. Deuschel, X. Guo and A. F. Ramirez. Quenched invariance principle for random walk in time-dependent balanced random environment $(2017+)$. To appear in Ann. Inst. H. Poincaré Probab. Statist.

A. Galves and E. Presutti. Edge fluctuations for the one-dimensional supercritical contact process. Ann. Probab. 15 (3), 1131-1145 (1987). MR893919.

O. Garet and R.e Marchand. Asymptotic shape for the contact process in random environment. Ann. Appl. Probab. 22 (4), 1362-1410 (2012). MR2985164.

T. E. Harris. Contact interactions on a lattice. Ann. Probability 2, 969-988 (1974). MR0356292.

M. R. Hilário, F. den Hollander, R. S. dos Santos, V. Sidoravicius and A. Teixeira. Random walk on random walks. Electron. J. Probab. 20, no. 95, 35 (2015). MR3399831.

F. den Hollander and R. S. dos Santos. Scaling of a random walk on a supercritical contact process. Ann. Inst. Henri Poincaré Probab. Stat. 50 (4), 1276-1300 (2014). MR3269994.

F. Huveneers and F. Simenhaus. Random walk driven by the simple exclusion process. Electron. J. Probab. 20, no. 105, 42 (2015). MR3407222.

T. M. Liggett. Stochastic interacting systems: contact, voter and exclusion processes, volume 324 of Grundlehren der Mathematischen Wissenschaften [Fundamental Principles of Mathematical Sciences]. Springer-Verlag, Berlin (1999). ISBN 3-540-65995-1. MR1717346.

T. Mountford and M. E. Vares. Random walks generated by equilibrium contact processes. Electron. J. Probab. 20, no. 3, 17 (2015). MR3311216.

F. Redig and F. Völlering. Random walks in dynamic random environments: a transference principle. Ann. Probab. 41 (5), 3157-3180 (2013). MR3127878.

R. S. dos Santos. Non-trivial linear bounds for a random walk driven by a simple symmetric exclusion process. Electron. J. Probab. 19, no. 49, 18 (2014). MR3217337. 